\title{
BMJ Open Suicide prevention for international students: a scoping review protocol
}

\author{
Sam McKay (D) , ${ }^{1,2}$ Angela Yuen Chun Li, ${ }^{1,2}$ Eleanor Bailey (D) ,1,2 \\ Michelle Lamblin, ${ }^{1,2}$ Jo Robinson ${ }^{1,2}$
}

To cite: McKay S, Li AYC, Bailey E, et al. Suicide prevention for international students: a scoping review protocol. BMJ Open 2022;12:e060266. doi:10.1136/ bmjopen-2021-060266

- Prepublication history and additional supplemental material for this paper are available online. To view these files, please visit the journal online (http://dx.doi.org/10.1136/ bmjopen-2021-060266).

Received 19 December 2021 Accepted 31 January 2022

Check for updates

(c) Author(s) (or their employer(s)) 2022. Re-use permitted under CC BY-NC. No commercial re-use. See rights and permissions. Published by BMJ.

${ }^{1}$ Orygen, The National Centre of Excellence in Youth Mental Health, Parkville, Victoria,

Australia

${ }^{2}$ Centre for Youth Mental Health, The University of Melbourne Faculty of Medicine Dentistry and Health Sciences, Melbourne, Victoria, Australia

Correspondence to

Dr Sam McKay;

samuel.mckay@orygen.org.au

\section{ABSTRACT}

Introduction The existing literature demonstrates that international students face a variety of stressors and barriers that can heighten the risk of suicide. However, up to now, no research has sought to summarise the available literature on the prevention strategies for suicide for international students in tertiary education. This document provides a scoping review protocol that aims to systematically chart and synthesise the published, unpublished and grey literature on the prevention strategies for suicide in the international student community.

Methods and analysis The enhanced six-stage methodological framework for scoping reviews of Arksey and 0'Malley will be used. Two main research questions guide the review: (1) What is the extent, range and nature of the evidence regarding suicide prevention for international students? and (2) What suicide prevention strategies are promising for targeting international students? Peer-reviewed and non-peer-reviewed articles, reports and policy documents will be eligible to be included in the review with no limits on publication date. Electronic searches of the CINAHL, ERIC, Medline, PsycInfo and ProQuest will be conducted to identify relevant academic publications. Grey literature searches will be undertaken on relevant databases as well as government and organisational websites. The reporting of the review will follow the guidelines of the Preferred Reporting Items for Systematic Reviews and Meta-Analyses Extension for Scoping Reviews. Criteria for evidence inclusion and exclusion will be used during literature screening and mapping. Screening and data charting of the published and grey literature will be conducted by three reviewers. Relevant stakeholders and experts will be consulted regarding the findings and their input will be integrated into the final report.

Ethics and dissemination The study will be disseminated through a peer-reviewed journal, conference presentations and consultations with relevant stakeholders in policy and professional settings. Ethical approval is not required for this review.

\section{BACKGROUND}

Suicide is a leading cause of death worldwide, ${ }^{1}$ and students enrolled in postsecondary education such as university are a key demographic at risk of suicide. ${ }^{2}$ Indeed, a meta-analysis on university students demonstrated that 12-month prevalence estimates
Strengths and limitations of this study

This will be the first scoping review to assess the prevention strategies for suicide in the international student community.

- The search strategy includes five electronic databases of peer-reviewed literature and a broad range of grey literature sources including government and other organisational websites.

- A quality appraisal of the published literature will be undertaken using the Standard Quality Assessment Criteria for Evaluating Primary Research Papers tool, but this will not be relevant to the grey literature.

- Relevant experts and stakeholders will be consulted throughout the scoping review process

for suicidal ideation, plans and attempts were $10.6 \%, 3.0 \%$ and $1.2 \%$, respectively. ${ }^{3}$ Data such as this highlights the importance of suicide prevention for this group and existing evidence suggests that postsecondary education is a setting where suicide prevention programmes can be delivered effectively. ${ }^{4-6}$ However, some groups may face a unique combination of risk factors and barriers that heighten the risk of suicide, ${ }^{7} 8$ requiring tailored suicide prevention strategies if interventions are to be effective. ${ }^{9}$

Postsecondary international students, referred to as international students for the remainder of this protocol, are such a group and make up a significant proportion of the student body in many countries. ${ }^{10}$ Although studying in another country may afford a variety of opportunities, ${ }^{11}$ international students face a unique set of stressors (eg, financial, language, cultural, discrimination, etc) ${ }^{12-14}$ that can negatively impact mental health, ${ }^{14}$ increase suicidal ideation ${ }^{71516}$ and even lead to death by suicide. ${ }^{17}$ Complicating matters, international students can face specific barriers such as lower mental health literacy and help-seeking intentions that can reduce engagement with support services, especially for suicidal ideation. ${ }^{8} 19$ This combination of factors may increase the risk of death by suicide for those who 
could otherwise receive help. ${ }^{18}$ Prevention strategies and programmes need to account for the unique barriers to reduce suicide risk in this group. ${ }^{78}$ In this document, a protocol for a scoping review is provided with the primary aim of identifying factors associated with effective suicide prevention strategies specifically targeting international students. The primary objectives of the review are:

1. To chart the extent, range and nature of available evidence on the prevention strategies for suicide in the international student community.

2. To identify gaps and limitations in the literature and provide future research recommendations to address them.

3. To guide suicide prevention policy and best practice guidelines for those working with international students.

\section{STUDY RATIONALE}

A scoping review was considered most appropriate for the current research for three main reasons. First, several reports $^{182021}$ and studies 2781922 have recognised that international students represent an important at-risk group for suicide. However, despite these findings and evidence that international students face a unique set of stressors and barriers that can heighten the risk of suicide ideation and death, ${ }^{78}$ no systematic review of interventions targeting this group has been conducted. Consequently, there is currently limited clarity on the extent, range and nature of evidence regarding prevention strategies for suicide in international students. Second, scoping reviews are ideally suited to understanding topics where grey literature is extensive. ${ }^{23}$ Suicide prevention efforts targeting international students are often only reported in the grey literature such as in government or institutional reports. ${ }^{182021}$ Accordingly, a scoping review offers an opportunity to provide a more comprehensive picture of the state of the literature on the topic. Third, topics that only have limited existing evidence, such as suicide prevention targeting international students, are well suited to a scoping review approach because opportunities for future research can be reported through the identification of existing knowledge gaps found across the full body of available evidence. ${ }^{23}$

This review follows the enhanced framework of scoping reviews ${ }^{23}$ that builds on the originally proposed methodology of Arksey and O'Malley. ${ }^{24}$ The framework incorporates six stages, each of which are detailed in the subsequent sections ${ }^{1}$ : identifying the research question, ${ }^{2}$ identifying relevant studies, ${ }^{3}$ selecting studies, ${ }^{4}$ mapping/charting the data, ${ }^{5}$ collating, summarising, reporting the results and $^{6}$ expert consultation. The reporting of the review will follow the guidelines of the Preferred Reporting Items for Systematic Reviews and Meta-Analyses (PRISMA) Extension for Scoping Reviews (PRISMA-ScR) ${ }^{25}$
METHODS

\section{Identifying the research question}

Research questions that guide scoping reviews are required to be broad enough to represent the extent of research on a specific topic, while clearly identifying the scope of enquiry. ${ }^{23}$ The population, concept, context (PCC) mnemonic is a recommended approach in the PRISMA-ScR guidelines for formulating research questions that provide a clear structure to scoping reviews. ${ }^{25}$ This approach was adopted when formulating the following research two questions:

1 . What is the extent, range and nature of the evidence regarding suicide prevention for international students?

2. What suicide prevention strategies are promising for targeting international students?

These questions will facilitate the mapping of the research conducted on suicide prevention specifically targeting international students, while also identifying existing knowledge gaps.

\section{Population}

International students are individuals enrolled in a postsecondary education programme at a college, university or other higher education provider in a country where they are not a citizen or permanent resident. ${ }^{10}$ The majority of postsecondary students are young people; however, age will not be controlled for, as adults of all ages can complete postsecondary education. ${ }^{10}$

\section{Concept}

Prevention strategies are the main concept pertinent to the research questions of this scoping review. Prevention strategies represent interventions, programmes or policies that aim to address a phenomenon, ${ }^{26} 27$ and in the case of this review, the key primary outcomes related to suicide (eg, death by suicide, suicide attempts, suicidal ideation). Prevention strategies differ by universal, indicated and selective intervention levels. ${ }^{28}$ Universal prevention strategies involve interventions that build skills and resilience or policies and legislation that protect individuals. ${ }^{28}$ Indicated prevention involves the referral of those with risk factors for suicide for assessment or treatment. ${ }^{28}$ Selective prevention approaches aim to prevent suicide among suicidal individuals. ${ }^{28}$

\section{Outcome}

The primary outcomes in this scoping review are death by suicide, suicide attempt and suicidal ideation. Death by suicide is death caused by intentional self-harm with an intent to die as a result of the behaviour. ${ }^{29}$ We will only include suicide deaths where the death is specifically determined to be suicide. A Suicide attempt is a nonfatal, self-directed, potentially injurious behaviour with an intent to die as a result of the behaviour. ${ }^{29}$ Suicidal ideation is thinking about, considering or planning suicide. $^{29}$

The methodology underpinning scoping reviews emphasises an iterative approach, ${ }^{23}$ accordingly, the 
research questions may be refined, or further questions added to the review, as familiarity with the literature increases within the research team.

\section{Context}

The context of this scoping review is postsecondary education settings worldwide. Given the recent COVID-19 pandemic and increasingly common use of online learning, ${ }^{10}$ for this review, postsecondary education settings can incorporate both local campuses as well as online environments, where students can engage with the educational services and staff. The worldwide focus was chosen because the literature on this topic is small and international students undertake postsecondary education across many countries, but face many similar challenges that can influence mental health across the different countries. ${ }^{131430}$

\section{Patient and public involvement}

There was no patient or public involvement in the development of this review protocol.

\section{Identifying relevant research}

A comprehensive systematic search strategy to identify relevant literature will be developed in consultation with a librarian following the guidance of the Joanna Briggs Institute (JBI) Reviewer's Manual for scoping reviews. ${ }^{31}$ The search strategy will target published, unpublished and grey literature. The strategy and rationale for any decisions regarding the search procedure will be included in the final manuscript.

The search strategy will follow the recommended three-step strategy for conducting scoping reviews. ${ }^{31}$ The first step involves an initial limited search of two relevant databases and analysis of keywords and phrases contained in the titles and abstracts of the retrieved papers, along with the index of terms and keywords describing the articles. The two databases for the initial search will be Medline (EBSCO) and PsycInfo (Ovid). A librarian will be consulted to develop search strings combining keywords, phrases and index terms employing Boolean operators (see online supplemental appendix A for proposed search strategy). The second step encompasses the adaptation and application of the search strings across all included databases: Medline (EBSCO), PsycInfo (Ovid), ERIC (EBSCO), CINAHL (EBSCO) and ProQuest Dissertations and Theses. Additionally, a selection of key academic journals will be identified by the review team and hand searched for relevant articles that may be missed during database searches. The third step comprises searching the evidence sources included in the review for any sources missed during step 2.

The review team is aware that there is an extensive body of potential sources of grey and unpublished literature on the topic of suicide prevention for international students. A systematic approach will be taken to identify potential grey and unpublished literature sources. The first step will involve a search of grey literature databases (eg,
Google Scholar, Open Grey, Trove-The National Library of Australia, the British Library, ResearchGate, Science. gov and UNESCO) using the keywords and phrases identified in the published articles. To identify governmental and organisational reports, and policy documents country-specific searches will be conducted on Google for the top four English-speaking destinations for international students (eg, USA, Canada, UK and Australia), which also represent the top five destinations worldwide with the only other country being China. ${ }^{32}$ The search will be limited to either using location-specific URL features (eg, Site:.au/.ca/.uk) or region-based searching when a location-specific URL is not available (eg, setting the search region to the USA because the USA does not have a country-specific URL ending). Additionally, the filetype:pdf search tool will be used to limit results to PDF files that are more likely to represent reports or other publications. Only the first 100 hits, sorted by relevance, in the grey literature databases will be screened because further screening will be unlikely to lead to additional relevant literature. ${ }^{33}$ Next, relevant evidence will be searched and screened including conference abstracts from leading conferences on suicide prevention and international students, theses and dissertations (eg, ProQuest Dissertations and Theses), preprints (eg, OSF Preprints). As a final step, academic and other experts from professional societies in the field will be contacted to identify any other available evidence that was not identified through the searches of published, unpublished and grey literature.

\section{Eligibility criteria}

Peer-reviewed and non-peer-reviewed articles, reports and policy documents will be eligible to be included in the review. We will endeavour to include relevant non-English publications but may exclude them at the time of full text review if translation is unfeasible. No limitation will be placed on the study design. However, where overlap is identified in either the participant samples or data sets of multiple publications, the source that provides the most information relevant to the review aims will be included. Review articles that provide novel insights will also be eligible for inclusion.

The search strategy will also follow the eligibility criteria based on the PCC mnemonic (including the primary outcomes for this scoping review) from the PRISMA-ScR guidelines. ${ }^{25}$ Table 1 lists the inclusion and exclusion criteria for the population (international university students), concept (prevention), outcome (suicide, suicide attempt, suicidal ideation) and context (postsecondary education settings). As per other sections of this protocol, the criteria may be refined as the scoping review team becomes more familiar with the literature on the subject. $^{23}$

\section{Selecting studies}

All search results will be imported into the systematic review screening tool Covidence, where any duplicates 
Table 1 Inclusion and exclusion criteria for study selection

Included Excluded

Population: international tertiary education students

- Sources that clearly denote that participants or a proportion of participants were international students

Sources that involve international students but do not include them as an independent group within any analyses

- Sources that do not clearly state if international students were part of the participant cohort

\section{Concept: prevention}

- Sources that studied interventions where the outcome was suicide-related (eg, death by suicide, suicide attempts, or suicidal ideation)

- Sources that provide recommendations for suicide prevention

Outcome: death by suicide, suicide attempt, suicidal ideation

- Sources that include death by suicide, suicide attempt or suicidal ideation as an outcome

- Sources that focus on non-suicide related mortality only or where the cause of death cannot be specifically attributed to suicide

\section{Context: postsecondary education settings}

- Sources that provide insight into suicide prevention for enrolled international students either in person or digitally within the host country or the students home country before or during their international educational programme

- Sources from any geographic region provided they are in English

will be removed. Following current best practice guidelines, the review team will meet at the start, during and at the end of each stage of selecting studies. ${ }^{23}$ During these meetings, any disagreements on inclusion will be discussed. The first step of the selection process will involve the review team independently applying the selection criteria to a random selection of 25 study titles and abstracts, followed by a meeting to discuss discrepancies and any necessary modifications for the criteria to ensure complete agreement. Next, two reviewers will apply the eligibility criteria to the titles and abstracts of retrieved sources in Covidence. All sources that are deemed ineligible for full-text review will be removed and the reason for their removal recorded. After this is completed, a separate reviewer will independently assess the excluded articles to confirm accuracy. If conflicts cannot be resolved, a fourth reviewer will be consulted to make a final decision. In the final stage, the remaining full-text publications will be independently reviewed by two separate reviewers and those deemed unsuitable will be excluded with the reason for exclusion recorded. In the case of disagreements, a discussion will be held until a consensus can be reached. If required, a third reviewer will be consulted until a final consensus can be reached on the final included and excluded articles.

As part of the review and selection process, a record of questions for discussion and associated verdicts will be kept and included as an online supplemental appendix with the final manuscript. A flow diagram following the PRISMA-ScR guidelines ${ }^{25}$ will be created and reported in the final manuscript to capture the reasons for exclusion of sources following the title and abstract and full-text review.

\section{Mapping/charting the data}

A data charting process will be conducted to extract relevant data from eligible sources for this scoping review. Data extraction tables will be used to record the data. The data charting tables will be based on previous systematic reviews of suicide prevention in education settings ${ }^{45}$ and align with the recommendations for data charting in the JBI reviewer's manual. ${ }^{31}$ The data that will be collected are study characteristics (eg, publication year and country), the overarching aim of the study or report, study design, study setting/context, population (eg, age, gender, ethnicity/country of origin and whether the student was in the host country during the study), outcome measures (eg, prevalence, suicide, suicidal ideation), any prevention strategy that was tested including the outcome assessed (eg, a programme aimed at increasing engagement with health services for suicidal ideation, etc), presence or absence of a control group, any risk factors examined (eg, correlates or predictors of suicide, suicide attempt or suicidal ideation), finding interpretations, recommendations for research, policy or practice and study limitations.

The data extraction tables will be piloted by two independent reviewers on a random selection of 10 publications before a discussion is undertaken on the completeness of the data chart and to assess the accuracy and consistency in the data extraction process. The data charting process is anticipated to be iterative and 
the initial data extraction tables may be refined during the pilot testing and charting periods. The full review team will undertake regular consultation during the data charting process and any disagreements will be addressed by a third reviewer. Contact will be made with the authors of relevant studies where further clarification is required regarding any evidence sources. The final manuscript will include a table with the data chart headings, and the charted data will be made publicly available.

Methodological quality will be assessed as part of the data charting process. Reliability, generalisability and credibility of the articles will be independently assessed using the Standard Quality Assessment Criteria for Evaluating Primary Research Papers tool. ${ }^{34}$ Scoring provides a total possible score of 28 for quantitative papers and 20 for qualitative papers. Scores will be converted to a percentage to create a consistent comparison metric with $>80 \%$ defined as strong, $70 \%-80 \%$ defined as good, $55 \%-69 \%$ considered adequate and $<55 \%$ considered limited. ${ }^{35}$ Inconsistencies in the assessment will be resolved through a discussion between reviewers.

\section{Collating, summarising and reporting the results}

All data from the selected articles will be collated, summarised and reported based on the aims of the scoping review and the PRISMA-ScR checklist guidelines for reporting results. ${ }^{25}$ The evidence sources will be aggregated and analysed using quantitative and qualitative methods. To provide an overview of the general state of the literature, the characteristics of the different included studies will be collated and presented. The characteristics will include publication dates, populations and samples, study location, used methodologies, interventions used (if any) and the type of evidence (eg, empirical article, report, etc). A narrative synthesis will be conducted on the prevention programmes for international student suicide. The synthesis will focus on intervention approaches and reported efficacy. As intervention programmes can address different levels, any reported interventions will be categorised as primary, secondary or tertiary. Additionally, the target of intervention will be reported (eg, suicidal ideation). Current recommendations from policies and reports will also be collated and reported as part of the review. Based on the narrative synthesis of the currently available evidence, research limitations and knowledge gaps, recommendations for future research, policy development and intervention opportunities will be provided. The collation and analysis of the data will be iterative, building on both discussions within the team and expert consultation. The full process underpinning the data synthesis and the rationale for any relevant decisions will be reported as part of the final manuscript.

\section{Expert consultation}

Expert consultation is a key component of scoping reviews, enhancing methodological rigour and applicability through engaging relevant stakeholders with expertise in research, policy and practice..$^{23}$ Consultations will occur with key national and international agencies involved in suicide prevention (eg, International Association for Suicide Prevention, and Australian Centre for Research Excellence in Suicide Prevention) and postsecondary education of international students (eg, The Council for International Education in Australia, and Study Melbourne). Additionally, experts from other relevant organisations, societies and research centres will be contacted. The preliminary findings from stage 5 will serve as the basis for the consultation process. There are four primary goals of the consultation: (1) to identify any sources of grey literature missed during the initial search, (2) to obtain additional insights and outlooks beyond those identified through the initial data analysis, (3) to identify opportunities for knowledge translation and (4) to present the preliminary findings the relevant stakeholders. The input from the consultations will be analysed, and where relevant, incorporated into the final review findings and recommendations.

\section{CURRENT STUDY STATUS}

At the time of publishing this protocol, preliminary searches of the relevant literature have been undertaken to commence the development of the search strategy and inform this protocol document.

\section{DISSEMINATION AND ETHICS}

The predominant aim of this scoping review is to identify the factors associated with effective suicide prevention strategies specifically targeting international students. The review will afford several important outcomes that will facilitate development in the field. Specifically, a clearer picture of the available evidence on the prevention strategies for suicide for international students will be established and enable the identification of current knowledge gaps and research limitations, along with recommendations for future research, policy and programme development. A scoping review approach is well suited to mapping the diversity of available literature in this area and this study will use the most up to date methodological approaches, which is a strength of the research. Furthermore, the stakeholder engagement that is part of the review process will offer opportunities for knowledge translation with experts who inform policy and practice in this area, further enhancing impact. Beyond stakeholder engagement, the review findings will be disseminated through a peer-reviewed academic journal, relevant conferences, and to other key experts and stakeholders in the field.

Twitter Sam McKay @samuel_McKay

Contributors SM, AYCL, EB, ML and JR: contributed to conception and design; contributed to planning for the acquisition, analysis and interpretation of data; critically revised the manuscript; gave final approval; and agreed to be accountable for all aspects of the work ensuring integrity and accuracy. SM drafted the manuscript.

Funding This project was funded by Future Generations Global. 
Competing interests None declared.

Patient consent for publication Not applicable.

Provenance and peer review Not commissioned; externally peer reviewed.

Supplemental material This content has been supplied by the author(s). It has not been vetted by BMJ Publishing Group Limited (BMJ) and may not have been peer-reviewed. Any opinions or recommendations discussed are solely those of the author(s) and are not endorsed by BMJ. BMJ disclaims all liability and responsibility arising from any reliance placed on the content. Where the content includes any translated material, BMJ does not warrant the accuracy and reliability of the translations (including but not limited to local regulations, clinical guidelines, terminology, drug names and drug dosages), and is not responsible for any error and/or omissions arising from translation and adaptation or otherwise.

Open access This is an open access article distributed in accordance with the Creative Commons Attribution Non Commercial (CC BY-NC 4.0) license, which permits others to distribute, remix, adapt, build upon this work non-commercially, and license their derivative works on different terms, provided the original work is properly cited, appropriate credit is given, any changes made indicated, and the use is non-commercial. See: http://creativecommons.org/licenses/by-nc/4.0/.

\section{ORCID iDs}

Sam McKay http://orcid.org/0000-0003-2700-5285

Eleanor Bailey http://orcid.org/0000-0003-4918-1618

\section{REFERENCES}

1 World Health Organization. Preventing suicide: a global imperative. Switzerland: World Health Organization, 2014.

2 Hong V, Busby DR, O'Chel S, et al. University students presenting for psychiatric emergency services: socio-demographic and clinical factors related to service utilization and suicide risk. J Am Coll Health 2020:1-10.

3 Mortier P, Cuijpers P, Kiekens G, et al. The prevalence of suicidal thoughts and behaviours among college students: a meta-analysis. Psychol Med 2018;48:554-65.

4 Wolitzky-Taylor K, LeBeau RT, Perez M, et al. Suicide prevention on College campuses: what works and what are the existing gaps? A systematic review and meta-analysis. J Am Coll Health 2020;68:419-29.

5 Robinson J, Calear AL, Bailey E. Suicide prevention in educational settings: a review. Australas Psychiatry 2018;26:132-40.

6 Black MH, Scott M, Baker-Young E, et al. Preventing suicide in post-secondary students: a scoping review of suicide prevention programs. Eur Child Adolesc Psychiatry 2021. doi:10.1007/s00787021-01858-8. [Epub ahead of print: 09 Aug 2021].

7 Taliaferro LA, Muehlenkamp JJ, Jeevanba SB. Factors associated with emotional distress and suicide ideation among international college students. J Am Coll Health 2020;68:565-9.

8 Clough BA, Nazareth SM, Day JJ, et al. A comparison of mental health literacy, attitudes, and help-seeking intentions among domestic and international tertiary students. Br J Guid Counc 2019;47:123-35.

9 Franklin JC, Ribeiro JD, Fox KR, et al. Risk factors for suicidal thoughts and behaviors: a meta-analysis of 50 years of research. Psychol Bull 2017;143:187-232.

10 OECD. Education at a glance 2020

11 Findlay AM, King R, Smith FM, et al. World class? an investigation of globalisation, difference and international student mobility. Transactions of the Institute of British Geographers 2012;37:118-31.

12 Golberstein E, Eisenberg D, Gollust SE. Perceived stigma and mental health care seeking. Psychiatr Serv 2008;59:392-9.
13 Zhang J, Goodson P. Predictors of international students' psychosocial adjustment to life in the United States: A systematic review. Int J Intercult Relat 2011;35:139-62.

14 Brunsting NC, Zachry C, Takeuchi R. Predictors of undergraduate international student psychosocial adjustment to US universities: a systematic review from 2009-2018. Int J Intercult Relat 2018;66:22-33.

15 Pérez-Rojas AE, Choi N-Y, Yang M, et al. Suicidal ideation among international students: the role of cultural, academic, and interpersonal factors. Couns Psychol 2021;49:673-700.

16 Wang KT, Wong YJ, Fu C-C, . Moderation effects of perfectionism and discrimination on interpersonal factors and suicide ideation. $J$ Couns Psychol 2013;60:367-78

17 McLaughlin JC, Gunnell D. Suicide Deaths in University Students in a UK City Between 2010 and 2018 - Case Series. Crisis 2021;42:171-8

18 Jamieson A. Findings into death of Zhikai Liu. Coroners Court of Victoria, 2019.

19 Nguyen M-H, Le T-T, Nguyen H-KT, et al. Alice in Suicideland: exploring the suicidal ideation mechanism through the sense of connectedness and help-seeking behaviors. Int J Environ Res Public Health 2021;18. doi:10.3390/ijerph18073681. [Epub ahead of print: 0104 2021].

20 Orygen. International student mental health and physical safety, 2020.

21 Forbes-Mewett H. Mental health and international students: issues, challenges and effective practice', Res. International Education Association of Australia (IEAA), 2019.

22 Servaty-Seib HL, Lockman J, Shemwell D, et al. Belongingness, and suicidal ideation. Suicide Life Threat Behav 2016;46:141-53.

23 Peters MDJ, Marnie C, Tricco AC, et al. Updated methodological guidance for the conduct of scoping reviews. JBI Evid Synth 2020;18:2119-26.

24 Arksey H, O'Malley L. Scoping studies: towards a methodological framework. Int J Soc Res Methodol 2005;8:19-32.

25 Tricco AC, Lillie E, Zarin W, et al. PRISMA extension for scoping reviews (PRISMA-ScR): checklist and explanation. Ann Intern Med 2018;169:467-73.

26 Anderson JK, Ford T, Soneson E, et al. A systematic review of effectiveness and cost-effectiveness of school-based identification of children and young people at risk of, or currently experiencing mental health difficulties. Psychol Med 2019;49:1-11.

27 Zalsman G, Hawton K, Wasserman D, et al. Suicide prevention strategies revisited: 10-year systematic review. Lancet Psychiatry 2016;3:646-59.

28 Mrazek PJ, Haggerty RJ. Reducing risks for mental disorders: frontiers for preventive intervention research. Washington, DC: National Academy Press, 1994.

29 Organization WH. International statistical classification of diseases and related health problems. 11th ed, 2020.

30 Cao C, Meng Q. A systematic review of predictors of international students' cross-cultural adjustment in China: current knowledge and agenda for future research. Asia Pacific Education Review, 2021.

31 Peters MD, Godfrey C, Mclnerney P. Chapter 11: scoping reviews (2020 version). In: JBI manual for evidence synthesis, 2020.

32 Institute of International Education. Project atlas: a quick look at global mobility trends, 2020.

33 Pham MT, Rajić A, Greig JD, et al. A scoping review of scoping reviews: advancing the approach and enhancing the consistency. Res Synth Methods 2014;5:371-85.

34 Kmet LM, Cook LS, Lee RC. Standard quality assessment criteria for evaluating primary research papers from a variety of fields, 2004.

35 Lee L, Packer TL, Tang SH, et al. Self-Management education programs for age-related macular degeneration: a systematic review. Australas J Ageing 2008;27:170-6. 